\title{
Efeito do teor e da dispersão de fino calcário na aderência substrato-matriz cimentícia
}

Influence of the content and dispersion of limestone fines in the bond strength of mortar-brick interfaces

\begin{abstract}
Eliane Betânia Carvalho Costa
Fábio Alonso Cardoso

Vanderley Moacyr John

\section{Resumo}

$\mathbf{E}$

ste artigo tem como objetivo avaliar o efeito do teor e da dispersão de partículas de fino calcário em substituição ao ligante para a aderência substrato-matriz cimentícia. Para tal, foram produzidas argamassas com $0 \%, 30 \%$ e $60 \%$ de substituição do teor de cimento por fino calcário, de granulometria inferior à do cimento; também foi avaliada a dispersão das partículas pela adição de $0,02 \%$ de dispersante à base de policarboxilato às argamassas aplicadas sobre blocos cerâmicos hidrofugados. O comportamento reológico das argamassas, medido por reometria rotacional, variou conforme o teor de fino calcário e o uso de dispersante. Apesar da redução das propriedades mecânicas, a substituição de $60 \%$ de ligante por finos calcários combinado ao uso de dispersante pode aumentar a aderência em 76\%. O acréscimo de aderência pode ser explicado pelo aumento da área de contato entre a argamassa e o substrato. Essa conclusão é de interesse prático, pois mostra que é possível reduzir a quantidade de ligante da matriz sem afetar sua resistência de aderência.
\end{abstract}

Palavras-chaves: Aderência. Fino calcário. Argamassa. Substrato. Dispersão. Ligante.

Eliane Betânia Carvalho Costa Universidade Tecnológica Federal do Curitiba - PR - Brasil

Fábio Alonso Cardoso Universidade de São Paulo São Paulo - SP - Brasil

Vanderley Moacyr John Universidade de São Paulo São Paulo - SP - Brasil

Recebido em 24/04/15 Aceito em 21/10/15

\section{Abstract}

This study investigates the effects of the content and dispersion of limestone fine particles on the bond strength of mortar-brick interfaces. Mortars with 0\%, 30\% and $60 \%$ replacement of binder by limestone fines with a particle size distribution lower than cement. We also evaluated particle dispersion by adding $0.02 \%$ polycarboxylate-based dispersing admixture to the mortars applied on the surfaces of clay bricks treated with a water repellent. The rheological behavior of the mortars varied depending on the particle size of the limestone fines used and the admixture added, measured by a rotational rheometer. Despite a reduction in the mechanical properties, the $60 \%$ replacement of of binder by limestone fines, associated with the use of dispersing admixture can increase the bond strength of the mortar-brick interface by $76 \%$. The increased adherence can be explained by the increased area or contact between substrate and mortar. The conclusion has practical implications, as it demonstrates that is possible to reduce the amount of matrix binder without changing bond strength.

Keywords: Bond strength. Limestone fine. Mortar. Substrate. Dispersing. Binder. 


\section{Introdução}

A aderência é um fenômeno de contato entre superfícies: quanto maior a proximidade destas, mais intensas as forças intermoleculares responsáveis por tal propriedade (KENDALL, 2001). Nos sistemas cimentícios a interface substrato-matriz cimentícia é o elo fraco por ser mais porosa que a própria matriz (OLLIVIER; MASO; BOURDETTE, 1995; SCRIVENER; CRUMBIE; LAUGESEN, 2004). Essa interface pode ser otimizada pela redução do teor de água, pela compactação, pela adição de partículas finas ou pelo grau de hidratação. A penetração de partículas e a precipitação de cristais no interior dos poros e/ou rugosidade do substrato poderá contribuir com a aderência (CARASEK, 1996; CARVALHO JUNIOR, 2005), no entanto tal contribuição irá depender da geometria dos poros e das partículas. É indispensável que as características reológicas e a distribuição de partículas da matriz sejam compatíveis com a superfície do substrato (porosidade/rugosidade), de modo a garantir seu total recobrimento (COSTA, 2014).

Além dos benefícios ambientais, a principal contribuição da adição de fino calcário em matrizes cimentícias é dada pelo efeito físico, uma vez que a quantidade de $\mathrm{CaCO}_{3}$ que pode reagir é limitada pelo teor de sulfato e alumina presente no cimento (DAMIDOT et al., 2011; MATSCHEI; LOTHENBACH; GLASSER, 2007). Segundo Scrivener, Crumbie e Laugesen (2004), a porosidade e a extensão da interface irão depender do tamanho mínimo das partículas presentes na matriz. A adição de partículas finas, menores que o cimento, à matriz poderá ser uma das alternativas para aumentar a área de contato (MOOSBERGBUSTNES; LAGERBLAD; FORSSBERG, 2004; LAWRENCE; CYR; RINGOT, 2003). Por apresentarem uma área específica elevada, poderão reduzir a porosidade da interface pelo preenchimento das irregularidades da superfície e dos vazios entre as partículas.

Estudos mostram que a substituição de $15 \%$ a $20 \%$ de ligante por fino calcário não altera significativamente a resistência à compressão mesmo quando não é feito o controle da área superficial e da distribuição granulométrica (BENTZ et al., 2009; NEHDI, 1996; TSIVILIS et al., 2000). Nenhuma evidência do efeito de elevados teores de substituição do ligante por finos calcários para a resistência de aderência entre argamassa e diferentes substratos, tais como agregados e blocos cerâmicos, foi registrada. Sabese, todavia, que a incorporação de partículas finas na matriz aumenta as forças de coesão entre as partículas, dificultando a dispersão do sistema (ROY et al., 1993). A aglomeração aumenta o tamanho efetivo das partículas, podendo comprometer o recobrimento da superfície pela matriz, porém tal fenômeno pode ser evitado/reduzido através do uso de aditivos dispersantes (UCHIKAWA; HANEHARA; SAWAKI, 1997)

Sendo assim, objetivo deste trabalho é avaliar o efeito do teor e da dispersão de partículas de um fino calcário em substituição ao cimento na aderência substrato-matriz cimentícia.

\section{Materiais e métodos}

\section{Substrato cerâmico}

Para a aplicação das argamassas foram utilizados blocos cerâmicos de vedação, do tipo selecta, de dimensões 9x19x39 cm. A topografia da superfície dos substratos foi medida pela técnica de interferometria a laser utilizando perfilômetro óptico (marca Bruker, modelo NP-Flex). Para a aquisição dos perfis 2D foram adotados os seguintes parâmetros:

(a) lente objetiva de 50x (Michelson) e multiplicador de $2 \mathrm{x}$, com passo de $\mathrm{x}=0,062 \mathrm{~mm}$ e $\mathrm{y}=0,047 \mathrm{~mm}$;

(b) velocidade de varredura $5 \mathrm{x}$; e

(c) pixels válidos de $2 \%$.

A Figura 1 apresenta um exemplo de um perfil representativo da superfície do bloco cerâmico estudado, numa magnificação de 100x. Observa-se que na prática nenhuma superfície é lisa; depende da escala de observação.

As forças capilares influenciam no transporte de fluidos (partículas e água) da argamassa para o substrato. Embora não seja comprovado seu efeito na formação da interface substrato-matriz cimentícias, pode ocorrer a redução da porosidade na região pelo arraste das partículas. A absorção de água do substrato também irá modificar a reologia da matriz na interface, restringindo seu espalhamento e levando à formação de defeitos interfaciais (HENDRICKX, 2009; BARBOSA, 2010). Além destes, se a perda de água da argamassa para o substrato for excessiva, podem ocorrer defeitos e microfissuras na interface devido à contração volumétrica (DETRICHÉ et al., 1984; DETRICHÉ; MASO, 1986). A fim de controlar tal efeito e garantir o maior espalhamento da matriz para o substrato e homogeneidade da interface, a superfície do substrato foi hidrofugada utilizando 
hidrofugante à base de uma emulsão de octiltrietoxissilano (AMERICAN..., 2015).

O hidrofugante foi diluído em água numa proporção 9:1 (água:hidrofugante) em relação à massa de água e aplicado na superfície do substrato cerâmico em três demãos com o auxílio do pincel. A secagem foi feita ao ar por um período de $24 \mathrm{~h}$ antes da aplicação da argamassa. A eficiência do tratamento foi calculada por ensaios de absorção capilar e medidas do ângulo de contato, comparando-se blocos cerâmicos com e sem aplicação de silano. Os ensaios de absorção capilar foram realizados conforme a metodologia descrita por Reda Taha, El-Dieb e Shrive (2005). A massa de água absorvida foi monitorada após 1, 5, 10, 30 e 60 min de imersão do bloco na água, sendo seis corpos de prova para cada situação. O ângulo de contato foi medido utilizando-se goniômetro (Krüss, DAS 10). As medidas foram feitas após $1 \mathrm{~min}$ de deposição de uma gota de água deionizada em temperatura de $25^{\circ} \mathrm{C}$ e volume constante de $8 \mu \mathrm{L}$. Foram feitas trinta leituras para cada tratamento em três blocos distintos.

Os resultados de absorção capilar e ângulo de contato indicam que o tratamento com silano tem efeito significativo na absorção capilar dos blocos cerâmicos. O tratamento com silano reduziu em $91 \%$ a capacidade de absorção capilar do bloco cerâmico após $60 \mathrm{~min}$ do contato com água; e o ângulo de contato foi de $35,4^{\circ}$ e de $100,1^{\circ}$ para a superfície dos blocos sem e com a aplicação de silano respectivamente. Assim, o tratamento superficial com silano foi eficiente para controlar os efeitos da absorção capilar do substrato.

\section{Materiais particulados: cimento, finos calcários e areia}

As argamassas foram moldadas utilizando-se CPV - ARI (Cauê), um fino calcário de granulometria inferior ao cimento - FC1 (Procarb 1 - Provale) e areia artificial de origem granítica. O CPV - ARI foi utilizado por ser um cimento com maior fração de clínquer e por não conter adições pozolânicas, que poderiam afetar a análise dos resultados.

A Tabela 1 apresenta a composição química do cimento e do fino calcário obtida por análise semiquantitativa de fluorescência de raios $\mathrm{X}$ (FRX) e análises químicas complementares.

A distribuição granulométrica das partículas de cimento e do fino calcário foi medida por difração a laser em via úmida (equipamento Malvern MSS Mastersizer com acessório Hydro 2000 UM). As partículas de finos calcários foram suspensas em água deionizada, e o cimento em álcool isopropílico, e dispersas em ondas ultrassônicas por $1 \mathrm{~min}$ em velocidade de rotação de $1.800 \mathrm{rpm}$ até obscurescência de $10 \%$. Foram realizadas quatro leituras, executadas num conjunto de dez repetições consecutivas. A distribuição granulométrica da areia foi obtida por peneiramento mecânico. A distribuição granulométrica e as propriedades físicas dos materiais são apresentadas na Figura 2 e na Tabela 2 respectivamente.

Figura 1 - Perfil topográfico da superfície do bloco cerâmico estudado, magnificação de 100x obtida pela técnica de interferometria a laser

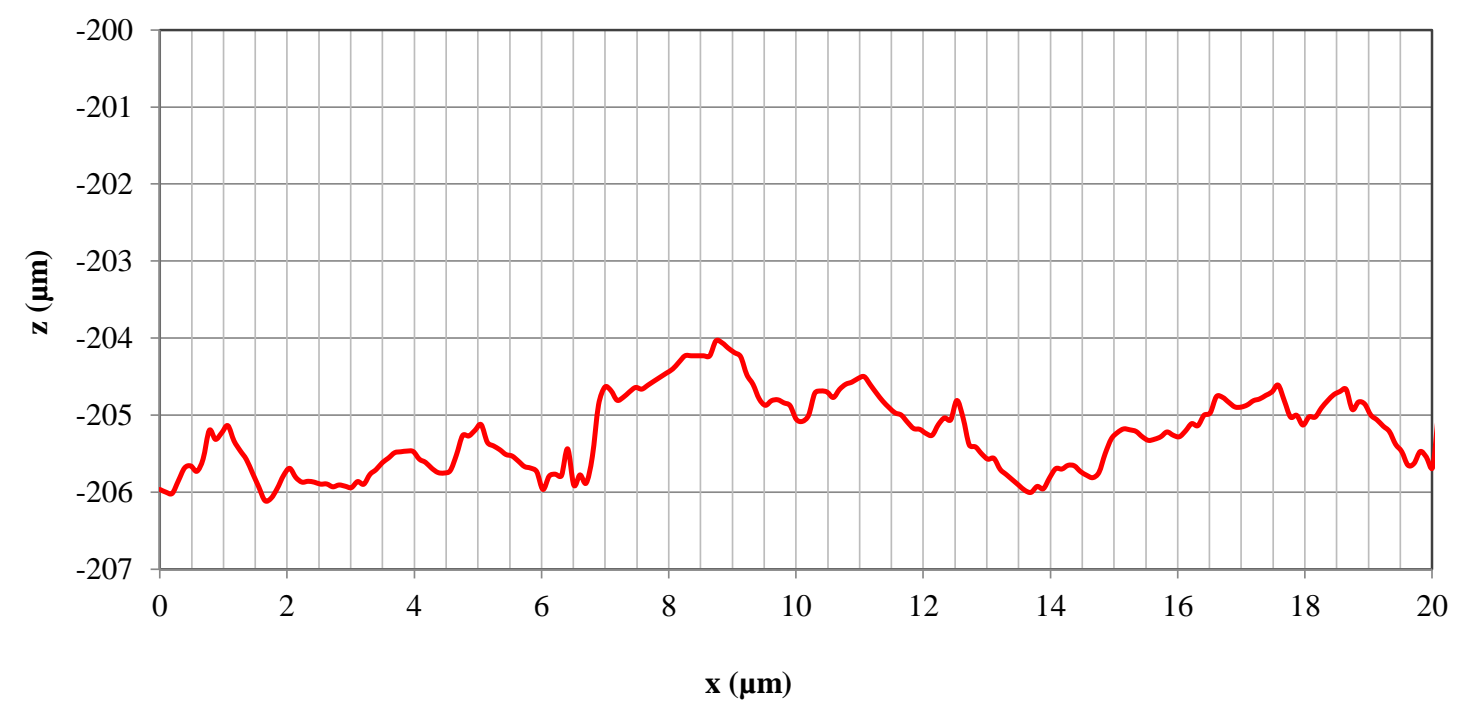


Tabela 1 - Composição química do cimento e do fino calcário utilizados

\begin{tabular}{c|c|c}
\hline Óxidos (\%) & Cimento & Fino calcário - FC1 \\
\hline $\mathrm{CaO}$ & 61,6 & 47,7 \\
$\mathrm{SiO}_{2}$ & 17,6 & 1,94 \\
$\mathrm{Al}_{2} \mathrm{O}_{3}$ & 4,98 & 0,59 \\
$\mathrm{Fe}_{2} \mathrm{O}_{3}$ & 2,62 & $<0,10$ \\
$\mathrm{MgO}$ & 1,99 & 5,99 \\
$\mathrm{Na}_{2} \mathrm{O}$ & 0,33 & 0,00 \\
$\mathrm{~K}_{2} \mathrm{O}$ & 1,00 & $<, 10$ \\
$\mathrm{SO}_{3}$ & 3,69 & $<, 10$ \\
Perda ao fogo - NBR - NM 18/04 & 4,87 & 43,1 \\
Óxido de cálcio livre - NBR - NM 13/04 & 2,29 & - \\
Resíduo insolúvel - NBR - NM 15/04 & 0,48 & 2,97 \\
$\mathrm{CO}_{2}$ (dióxido de carbono) - NBR - NM 20/04 & 2,67 & 41,6 \\
Equivalente alcalino em massa \% de Na $\mathrm{O}^{1}$ & 0,99 & - \\
\hline
\end{tabular}

Nota: ${ }^{1}$ equivalente alcalino em $\mathrm{Na}_{2} \mathrm{O}=\% \mathrm{Na}_{2} \mathrm{O}+0,658 \times \% \mathrm{~K}_{2} \mathrm{O}$. O ensaio foi realizado seguindo as diretrizes gerais da ISO/FDIS 29581-2:2009(E) (INTERNATIONAL...., 2009) - Cement - Test methods - Part 2: Chemical analysis by X-ray fluorescence e o espectrômetro de fluorescência de raios X (Panalytical - Minipal Cement), a partir de pastilhas fundidas, utilizando-se fundentes à base de mistura de tetraborato de lítio/metaborato de lítio marca Maxxiflux (66,67\% de Li2B4O7; 32,83\% de LiBO2 e 0,50\% de LiBr), com proporção de 1,0:6,75 (amostra:fundente) em g.

Figura 2 - Distribuição granulométrica dos materiais particulados obtidos por técnicas de difração a laser para o cimento e o fino calcário (FC1) e peneiramento mecânico a seco para areia.

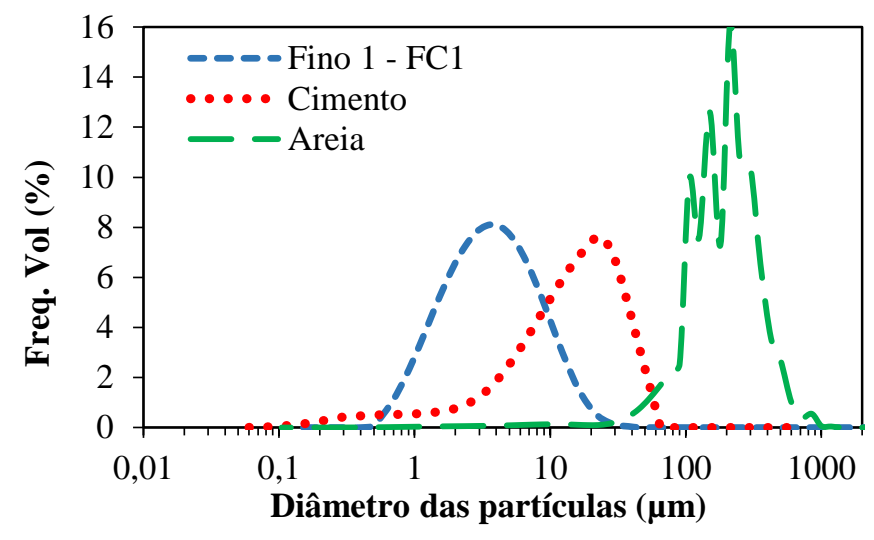

Tabela 2 - Propriedades físicas dos materiais sólidos utilizadas para confecção das argamassas

\begin{tabular}{|c|c|c|c|}
\hline Propriedades físicas & Cimento & $\begin{array}{c}\text { Fino calcário } \\
\text { FC1 }\end{array}$ & Areia \\
\hline Densidade $\left(\mathrm{g} / \mathrm{cm}^{3}\right)^{\mathrm{a}}$ & 3,04 & 2,63 & 2,82 \\
\hline Área superficial específica BET $\left(\mathrm{m}^{2} / \mathrm{g}\right)^{\mathrm{b}}$ & 1,87 & 2,97 & - \\
\hline Área superficial volumétrica BET $\left(\mathrm{m}^{2} / \mathrm{cm}^{3}\right)^{\mathrm{c}}$ & 5,69 & 7,81 & - \\
\hline Área superficial por difração laser ${ }^{\mathrm{d}}$ & 0,39 & 0,89 & - \\
\hline Fator de forma ${ }^{\mathrm{e}}$ & 4,8 & 3,3 & - \\
\hline D10 ${ }^{\mathrm{f}}$ & $2,87 \mu \mathrm{m}$ & $1,39 \mu \mathrm{m}$ & $102,27 \mu \mathrm{m}$ \\
\hline D50 ${ }^{\mathrm{f}}$ & $16,27 \mu \mathrm{m}$ & $3,97 \mu \mathrm{m}$ & $216,79 \mu \mathrm{m}$ \\
\hline D90 ${ }^{\mathrm{f}}$ & $39,25 \mu \mathrm{m}$ & $10,94 \mu \mathrm{m}$ & $406,94 \mu \mathrm{m}$ \\
\hline
\end{tabular}

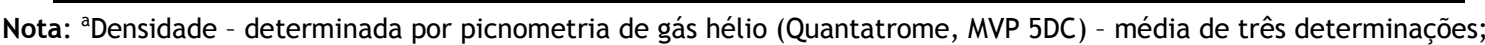
bárea superficial específica (BET) - ASTM D 3663/03 (AMERICAN..., 2015)- determinada por BET multiponto com adsorção de nitrogênio (ASAP 2010, Micromeritics);

cárea superficial volumétrica (VSA) é obtida pela área superficial específica dividida pela densidade; e dárea superficial específica estimada pela difração a laser (Mastersizer MSS, Malvern).

efator de forma é obtido pela relação entre a área superficial obtida pelo BET e a área obtida pela difração a laser.

fD10, D50 e D90 - indica o diâmetro em que a porcentagem de partículas é inferior ao valor correspondente, 10\%, $50 \%$ e $90 \%$ respectivamente. 
Os resultados mostram que o fino calcário utilizado apresenta partículas menores que o cimento. Pode ser visto na Tabela 2 que o fino calcário FC1 apresenta partículas com tamanho máximo de partículas igual a $20 \mu \mathrm{m}$ e pelo menos $90 \%$ das partículas menores que $11 \mu \mathrm{m}$ (D90). A Tabela 2 também mostra que a área superficial volumétrica (VSA) do $\mathrm{FC} 1$ foi $37 \%$ superior à do cimento. $\mathrm{O}$ fator de forma foi determinado dividindo a área superficial específica BET pela área superficial estimada pela distribuição granulométrica, assumindo que as partículas são esferas sólidas e perfeitamente lisas (HUNGER; BROUWERS, 2009). O fator de forma do cimento e do fino calcário foram 4,8 e 3,3 respectivamente. Tal resultado indica que as partículas do fino calcário são mais esféricas e/ou menos rugosas em comparação ao cimento.

A areia utilizada apresentou elevada quantidade de partículas inferiores a $75 \mu \mathrm{m} \quad(34,77 \%)$. A fissuração das argamassas devido ao elevado teor de materiais pulverulentos da areia utilizada poderia influenciar na avaliação das propriedades mecânicas e na aderência. Cuidados com a cura e controle de absorção do substrato foram tomados a fim de evitar tal efeito. Além disso, o teor de areia foi mantido constante em todas as composições estudadas e foi feita uma homogeneização prévia do material antes de ser adicionado à mistura com outros sólidos.

\section{Proporção das argamassas}

Foram estudadas três composições, sendo uma de referência (cimento e areia) com traço convencional e duas com substituição do cimento por FC1 em teores de $30 \%$ e $60 \%$ em volume. Para avaliar o efeito da dispersão das partículas, as três argamassas também foram moldadas com $0,02 \%$ de dispersante à base de policarboxilato (Melflux ${ }^{\circledR}$
$2651 \mathrm{~F}$, Basf) em relação à massa total de sólidos. $\mathrm{O}$ teor de água (deionizada) foi mantido constante e igual a 16,5\% em relação à massa total de sólidos em todas as composições estudadas. Esse teor foi estabelecido após um estudo prévio a fim de garantir elevada fluidez da argamassa, facilitando o espalhamento dela sobre o substrato. Os traços das argamassas bem como teor de dispersante, a relação água/cimento e o consumo de cimento são apresentados na Tabela 3.

\section{Mistura e avaliação do comportamento reológico das argamassas}

A mistura das argamassas foi feita em reômetro rotacional do tipo planetário com velocidade de rotação de 126,5 rpm por $5 \mathrm{~min}$. Os materiais sólidos foram previamente homogeneizados, e a água adicionada ao pó com vazão controlada de $128 \mathrm{~g} / \mathrm{s}$ a partir de $30 \mathrm{~s}$. O aditivo dispersante, quando utilizado, foi juntado aos sólidos antes da mistura.

Após a mistura, a argamassa foi submetida a um ciclo de cisalhamento, para avaliar o comportamento reológico e a eficiência do processo de mistura (DE FRANÇA, 2013). Durante o ciclo de cisalhamento, a velocidade rotacional era aumentada de 6,3 a 316,3 rpm, acelerando e desacelerando em patamares de $5 \mathrm{~s}$. A tensão de escoamento $\left(\tau_{0}\right)$ e a viscosidade plástica ( $\eta$ ) foram obtidas pela curva torque vs. velocidade após o ciclo de desaceleração.

Após o ciclo de cisalhamento, determinaram-se a densidade e o teor de ar das argamassas conforme os procedimentos estabelecidos pela NBR 13278 (ABNT, 2005), e foram moldados os corpos de prova para avaliação do desempenho mecânico das argamassas e da aderência.

Tabela 3 - Traço das argamassas estudadas indicando teor de dispersante, relação água/cimento (a/c) e consumo de cimento

\begin{tabular}{|c|c|c|c|c|c|}
\hline Argamassa & $\begin{array}{l}\text { Traço em volume } \\
\text { (cimento:fino } \\
\text { calcário:areia) }\end{array}$ & $\begin{array}{c}\text { Traço em massa } \\
\text { (cimento:fino } \\
\text { calcário:areia) }\end{array}$ & $\begin{array}{c}\text { Teor de } \\
\text { dispersante } \\
(\%)\end{array}$ & $\mathbf{a} / \mathbf{c}$ & $\begin{array}{c}\text { Consumo de } \\
\text { cimento } \\
\left(\mathrm{kg} / \mathrm{m}^{3}\right) \\
\end{array}$ \\
\hline$\overline{\mathrm{REF}}$ & $1: 0: 3$ & $1: 0: 2,8$ & 0,00 & 0,68 & 593,0 \\
\hline F130 & $1: 0,4: 4,3$ & $1: 0,4: 4,0$ & 0,00 & 0,96 & 415,1 \\
\hline F160 & $1: 1,5: 7,5$ & $1: 1,3: 7,0$ & 0,00 & 1,67 & 237,2 \\
\hline REF-D & $1: 0: 3$ & $1: 0: 2,8$ & 0,02 & 0,68 & 593,0 \\
\hline F130-D & $1: 0,4: 4,3$ & $1: 0,4: 4,0$ & 0,02 & 0,96 & 415,1 \\
\hline F160-D & $1: 1,5: 7,5$ & $1: 1,3: 7,0$ & 0,02 & 1,67 & 237,2 \\
\hline
\end{tabular}

Nota: o teor de água foi mantido constante e igual a 16,5\% em relação à massa total de sólidos para todas as argamassas estudadas. 


\section{Determinação do desempenho mecânico das argamassas}

O desempenho mecânico das argamassas foi avaliado pelos ensaios de módulo de elasticidade dinâmico e resistência à tração por compressão diametral, conforme recomendações da NBR 15630 (ABNT, 2008) e NBR 7222 (ABNT, 2011) respectivamente. Foram moldados seis corpos de prova cujas dimensões $(\varnothing=50 \mathrm{~mm}$ x $25 \mathrm{~mm})$ foram estabelecidas conforme Rocco et al. (1999), que determinaram as relações entre diâmetro e espessura que geram resultados mais próximos da tração direta do material.

A argamassa foi aplicada em camada única, e os corpos de prova adensados em mesa vibratória de bancada (MVL máquinas vibratórias, mod. MJ2008), na frequência de $50 \mathrm{~Hz}$ durante 10 s. A superfície foi nivelada com o auxílio de espátula metálica, e após $24 \mathrm{~h}$ os corpos de prova foram retirados do molde. Então a foram colocados em embalagens plásticas para garantir as mesmas condições de temperatura $\left(23 \pm 2{ }^{\circ} \mathrm{C}\right)$ e umidade $(\mathrm{UR}=100 \%)$ durante 13 dias. Após esse período foram colocados em estufa com circulação de ar em temperatura de $50{ }^{\circ} \mathrm{C}$ durante $24 \mathrm{~h}$.

O módulo de elasticidade dinâmico foi determinado utilizando-se o aparelho de ultrassom (PUNDIT, CNS Electronics) com transdutores de $200 \mathrm{KHz}$ e superfície geométrica de $3,14 \mathrm{~cm}^{2}$ (ABNT, 2008). O ensaio de resistência à tração por compressão diametral foi executado em máquina de ensaios universal (Instron 5569) segundo recomendações da NBR 7222 (ABNT, 2011). Ambos os ensaios foram realizados aos 14 dias.

\section{Determinação da resistência de aderência à tração}

A Figura 3 apresenta um detalhe da produção e preparo dos corpos de prova para a execução do ensaio de resistência de aderência à tração. Para cada situação foram moldados no mínimo 20 corpos de prova com o auxílio de um molde cilíndrico de PVC, com $50 \mathrm{~mm}$ de diâmetro e $20 \mathrm{~mm}$ de espessura, colocado sobre a superfície dos blocos cerâmicos. O procedimento de moldagem foi adotado a fim de eliminar a influência do corte nos valores de resistência de aderência à tração (COSTA, 2007) e facilitar a execução dos ensaios. Não foi elaborado um estudo comparativo entre o método adotado e o previsto pela NBR 13528 (ABNT, 2010), partindose do pressuposto de que as variações decorrentes do método seriam as mesmas para todos os sistemas estudados. As argamassas foram aplicadas sobre os blocos cerâmicos em camada única, vibradas conforme procedimento descrito anteriormente. $\mathrm{O}$ excesso de argamassa foi removido com o auxílio de espátula imediatamente após a moldagem. Após $24 \mathrm{~h}$ os corpos de prova foram desmoldados e permaneceram em câmara seca, em embalagens plásticas, para garantir as mesmas condições de temperatura e umidade durante 13 dias. Antes da colagem das pastilhas foi feita limpeza na superfície da argamassa para a retirada de partículas soltas. Foi utilizada uma massa de poliéster, curada por $24 \mathrm{~h}$ ao ar. $\mathrm{O}$ ensaio foi realizado com o dinamômetro de tração Dynatest DTE 5000.

\section{Resultados e discussão Comportamento reológico das
argamassas}

As curvas de mistura mostraram que o potencial de aglomeração das partículas de FC1 foi maior que a tendência de aglomeração das partículas de cimento, pelo menos nos primeiros minutos de contato com a água, provocando o aprisionamento de maior quantidade de água dentro dos aglomerados. A Figura 4 apresenta os resultados do ciclo de cisalhamento das argamassas (a) sem e (b) com o uso de aditivo dispersante.

As matrizes com substituição do ligante pelo fino calcário FC1 aumentaram os níveis de torque nas velocidades mais elevadas em comparação com a argamassa de referência para ambos os teores utilizados, sendo o efeito mais acentuado com o teor mais elevado (60\% FC1). Tal comportamento pode ser explicado pelo acréscimo de $37 \%$ da área superficial do FC1 em relação à do cimento. Isso diminuiu a distância entre as partículas e a mobilidade da matriz, resultando numa argamassa menos fluida, como pode ser constatado na Figura 4 a pelo aumento do nível do torque e de viscosidade. Na Figura $4 b$ observa-se que a adição de aditivo dispersante reduziu os níveis de torque em todas as argamassas estudadas, aumentando a fluidez da mistura e, consequentemente, sua dispersão. 
Figura 3 - Detalhe da produção e preparo do conjunto argamassa-substrato para a realização do ensaio de resistência de aderência à tração - Dynatest DTE - 5000

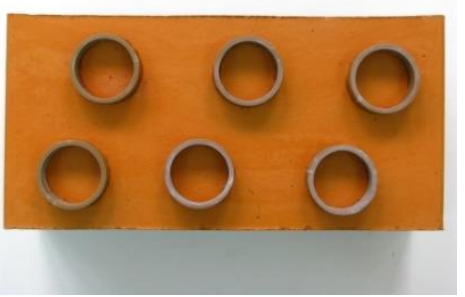

(a) Disposição dos moldes cilíndricos sobre os substratos

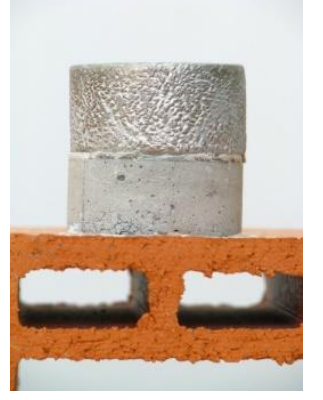

(d) Vista lateral do corpo de prova após a colagem da pastilha

Fonte: Costa (2014).

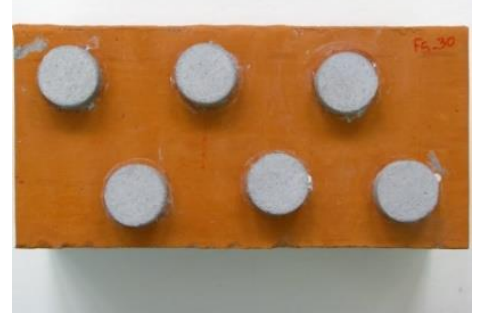

(b) Sistema argamassa-substrato após moldagem

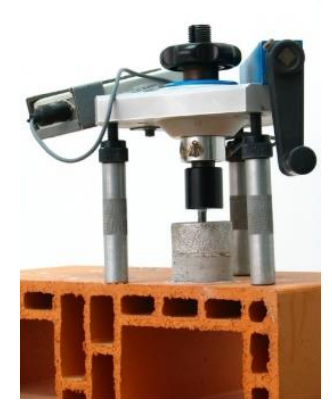

(e) Acoplamento pastilhaequipamento

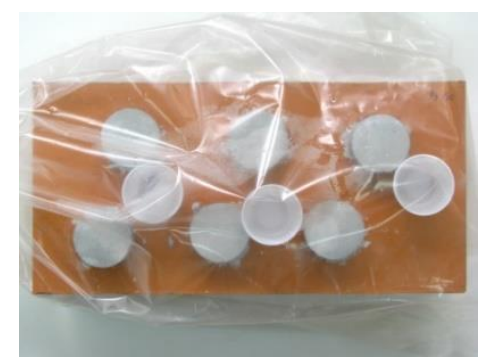

(c) Condicionamento do conjunto por 13 dias

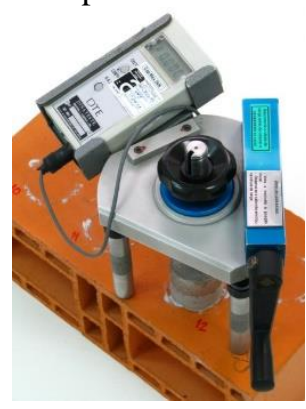

(f) Dinamômetro utilizado para a execução do ensaio

Figura 4 - Ciclo de desaceleração das argamassas estudadas (a) sem e (b) com o uso de aditivo dispersante

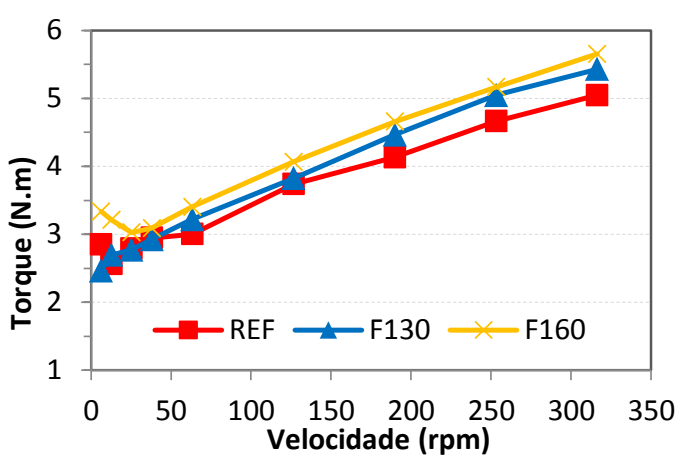

(a)

\section{Propriedades mecânicas das argamassas}

A análise de variâncias (ANOVA) ${ }^{1}$ do efeito do teor de fino calcário e de dispersante nas propriedades mecânicas das argamassas é apresentada na Tabela 4.

Os resultados mostram que o teor de fino calcário interfere significativamente no módulo de elasticidade e na resistência à tração das

${ }^{1}$ Técnica da análise de variâncias (ANOVA) utilizando-se o programa Statistic 6.0 da Statsoft $\circledast$. Os valores de $F_{\text {calculados }}\left(F_{\text {cal }}\right)$ foram comparados com os valores tabelados $\left(F_{\text {tab }}\right)$ a um nível de significância de $5 \%$. $O$ valor de $F_{\text {tab }}$ é igual a $F_{a=0,05}\left(v_{1}\right.$ e $\left.v_{2}\right)$, sendo $\mathrm{v}_{1}$ e $\mathrm{v}_{2}$ os graus de liberdade do efeito avaliado e do resíduo.

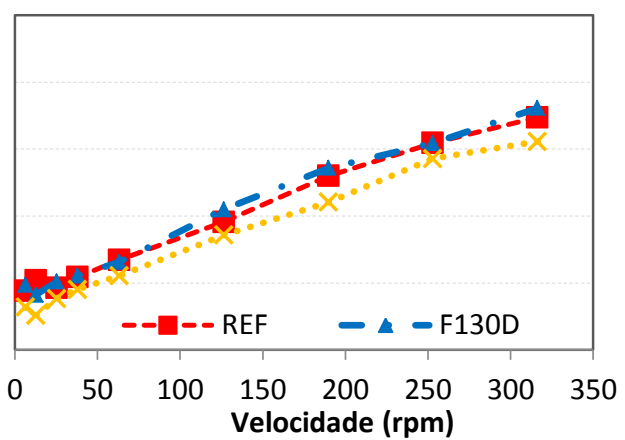

(b)

argamassas. O aumento do teor de finos provocou a redução das propriedades mecânicas das argamassas devido ao efeito de diluição, pois, com a redução do teor de cimento, menor o volume de produtos hidratados (LAWRENCE; CYR; RINGOT, 2003), e, como o teor de água foi mantido constante, houve aumento da porosidade. Resultados semelhantes têm sido relatados na literatura para concretos com substituição do cimento por finos calcários (NEHDI, 1996; TSIVILIS et al., 2000; CAMILETTI; SOLIMAN; NEHDI, 2012). 
Tabela 4 - Análise de variância da influência do teor de fino calcário e dispersante no módulo de elasticidade dinâmico e resistência à tração por compressão diametral das argamassas estudadas

\begin{tabular}{|c|c|c|c|c|c|c|c|}
\hline Fonte de variação & Propriedade & SQ & GL & MQ & Fcal & Ftab & Resultado \\
\hline Teor de fino calcário (A) & \multirow{5}{*}{$\begin{array}{l}\text { Módulo de } \\
\text { elasticidade } \\
\text { dinâmico }\end{array}$} & 2384,16 & 2 & 1192,08 & 2151,59 & 3,20 & $\mathrm{~S}$ \\
\hline Teor de dispersante (B) & & 60,23 & 1 & 60,23 & 108,70 & 4,05 & $\mathbf{S}$ \\
\hline$(\mathrm{A}) *(\mathrm{~B})$ & & 5,00 & 2 & 2,50 & 4,51 & 3,20 & $\mathrm{~S}$ \\
\hline Erro & & 23,27 & 42 & 0,55 & - & - & - \\
\hline Total & & 2472,66 & 47 & - & - & - & - \\
\hline Teor de fino calcário (A) & \multirow{5}{*}{$\begin{array}{l}\text { Resistência à } \\
\text { tração por } \\
\text { compressão } \\
\text { diametral }\end{array}$} & 66,50 & 2 & 33,25 & 262,21 & 3,20 & $\mathbf{S}$ \\
\hline Teor de dispersante (B) & & 0,22 & 1 & 0,22 & 1,73 & 4,05 & NS \\
\hline$(\mathrm{A}) *(\mathrm{~B})$ & & 0,46 & 2 & 0,23 & 1,81 & 3,20 & NS \\
\hline Erro & & 5,32 & 42 & 0,13 & - & - & - \\
\hline Total & & 72,5 & 47 & - & - & - & - \\
\hline
\end{tabular}

Nota: Legenda:

$\mathrm{SQ}=$ soma dos quadrados;

$\mathrm{GL}$ = grau de liberdade;

$M Q$ = média dos quadrados;

$\mathrm{F}=$ parâmetro de Fisher para o teste de significância dos efeitos;

$\mathrm{S}=$ significativo; $\mathrm{e}$

NS = não significativo.

A adição de dispersante reduziu significativamente o módulo de elasticidade em relação às argamassas sem o uso do aditivo. O teste de comparação múltipla de médias (Teste de Duncan) indicou a distinção de seis grupos distintos. Essa redução do módulo provavelmente ocorreu devido ao aumento da porosidade ocasionado pelo aumento do teor de ar incorporado na argamassa com aditivo dispersante no estado fresco, como mostra a Tabela 5. A substituição de ligante por finos reduz o módulo de elasticidade do material. No caso de argamassas de revestimentos, o aumento da deformabilidade reduz o aparecimento de fissuras (FIORITO, 2009).

Embora o teor de dispersante tenha reduzido o valor da resistência à tração, de acordo com a análise estatística esta não foi significativa. Comparando as médias entre os pares de argamassa com e sem a presença de dispersante, o teste de Duncan indicou diferença somente entre as argamassas F130 e F130-D.

\section{Resistência de aderência à tração}

A Figura 5 apresenta os resultados de resistência de aderência à tração aos 14 dias das argamassas sem e com o uso de aditivo dispersante aplicadas sobre os blocos cerâmicos hidrofugados. O coeficiente de variação dos resultados situa-se ente $19 \%$ e 34\%, valores típicos deste ensaio (COSTA, 2007). Todos os corpos de prova apresentaram ruptura na interface argamassa-substrato.

A análise estatística indicou que ambas as variáveis estudadas, teor de fino e de dispersante, interferem significativamente na resistência de aderência à tração, como mostra a Tabela 6. De acordo com o teste de Duncan, a argamassa F160 difere-se das demais argamassas estudadas.

A substituição de $30 \%$ do ligante pelo fino calcário na argamassa sem dispersante propiciou aumento de $18 \%$ da resistência de aderência em relação à argamassa de referência, e para o teor de $60 \%$ houve redução da aderência.

$\mathrm{O}$ uso de aditivo dispersante aumentou significativamente a resistência de aderência para todas as argamassas estudadas, com aumento médio de 29\%; no entanto, não melhorou as propriedades mecânicas das argamassas. Tal efeito também foi constatado por Antunes (2005), ao comparar argamassas sem e com o uso de dispersante. A autora verificou acréscimo médio da resistência de aderência de $21 \%$ das argamassas com o aditivo.

A Figura 6 mostra que há uma relação entre a resistência de aderência e a tensão de escoamento das argamassas. As argamassas com dispersante apresentam menor tensão de escoamento, isto é, são mais fluidas, o que tende a favorecer $\mathrm{o}$ espalhamento delas sobre o substrato, e consequentemente maior a resistência de aderência. O dispersante também promove individualização das partículas (ROY et al., 1993) - tal efeito pode ser constatado na argamassa F160-D. O uso de dispersante associado ao elevado teor de fino calcário propiciou um ganho de resistência de aderência de $76 \%$ e de $12 \%$ em relação à F160 e à de REF respectivamente. Tais resultados mostram o enorme potencial de utilização de partículas finas em teores elevados para a obtenção de uma aderência satisfatória. 
Tabela 5 - Propriedades das argamassas no estado fresco e endurecido

\begin{tabular}{l|c|c|c|c|c|c}
\hline \multirow{2}{*}{ Argamassa } & \multicolumn{4}{|c|}{ Estado fresco } & \multicolumn{2}{c}{ Estado endurecido } \\
\cline { 2 - 7 } & $\begin{array}{c}\text { Densidade } \\
\left(\mathbf{g} / \mathbf{c m}^{\mathbf{3}}\right)\end{array}$ & $\begin{array}{c}\text { Teor de } \\
\mathbf{a r}(\boldsymbol{\%})\end{array}$ & $\boldsymbol{\tau}_{\mathbf{0}} \mathbf{( \mathbf { N . m } )}$ & $\boldsymbol{\eta} \mathbf{( N . m )}$ & $\mathbf{E}(\mathbf{G P a})$ & $\mathbf{R t}(\mathbf{M P a})$ \\
\hline REF & 2,20 & 0,0 & 2,62 & 0,008 & $33,4 \pm 0,7$ & $4,4 \pm 0,4$ \\
F130 & 2,20 & 0,9 & 2,55 & 0,010 & $27,4 \pm 0,8$ & $3,7 \pm 0,4$ \\
F160 & 2,20 & 0,5 & 2,99 & 0,009 & $16,4 \pm 0,5$ & $1,7 \pm 0,2$ \\
REF-D & 2,10 & 2,5 & 1,82 & 0,009 & $31,7 \pm 1,3$ & $4,5 \pm 0,4$ \\
F130-D & 2,10 & 2,2 & 1,81 & 0,009 & $24,3 \pm 0,6$ & $3,4 \pm 0,3$ \\
F160-D & 2,10 & 2,1 & 1,55 & 0,009 & $14,4 \pm 0,4$ & $1,6 \pm 0,2$ \\
\hline
\end{tabular}

Figura 5 - Resistência de aderência à tração das argamassas estudadas aos 14 dias

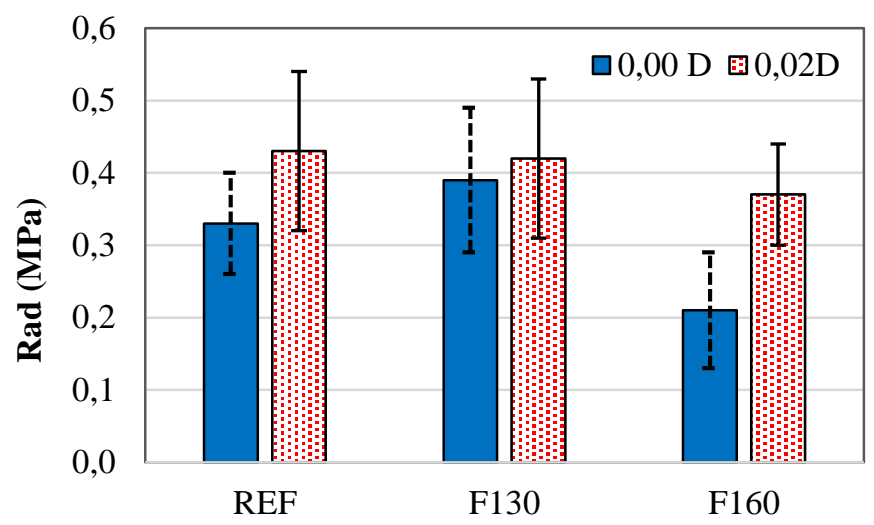

Argamassas estudadas

Tabela 6 - Análise de variância da influência do teor de fino calcário e de dispersante na resistência de aderência à tração argamassa-substrato.

\begin{tabular}{lc|c|c|c|c|c}
\hline \multicolumn{1}{c|}{ Fonte de variação } & SQ & GL & MQ & Fcal & Ftab & Resultado \\
\hline Teor de fino calcário (1) & 0,42 & 2 & 0,21 & 18,42 & 3,06 & S \\
Teor de dispersante (2) & 0,23 & 1 & 0,23 & 20,05 & 3,91 & S \\
$(1)^{*}(2)$ & 0,12 & 2 & 0,06 & 5,29 & 3,06 & S \\
Erro & 1,63 & 143 & 0,01 & - & - & - \\
Total & 2,40 & 148 & - & - & - & - \\
\hline
\end{tabular}

Figura 6 - Relação entre a resistência de aderência à tração e tensão de escoamento das argamassas estudadas

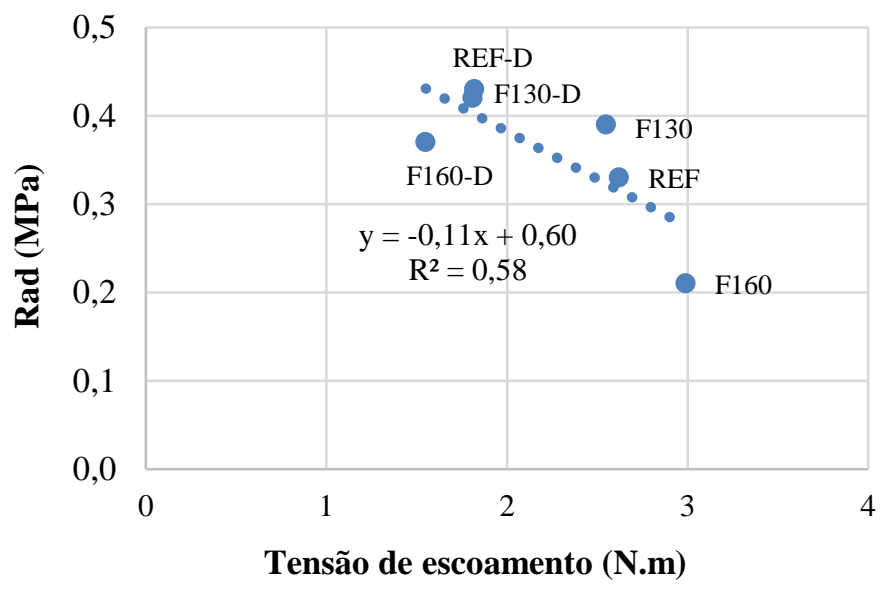


Tomando por exemplo o perfil topográfico do bloco cerâmico estudado obtido por interferometria a laser numa magnificação de 100x e o diâmetro das partículas (D10) de cimento e do fino calcário mais fino (FC1), obtido pela granulometria a laser, simulou-se a deposição delas sobre a superfície do substrato num sistema monodisperso de cimento (Figura 7) e em outro polidisperso, constituído por cimento e fino calcário FC1 (Figura 8).
As partículas mais finas tendem a migrar para a superfície do substrato (HU; STROEVEN, 2004; SCRIVENER; CRUMBIE; LAUGESEN, 2004), podendo ocasionar o aumento do contato da matriz com o substrato. Observa-se na Figura 8 que, embora o uso de FC1 proporcione melhor recobrimento da superfície do substrato, este não é totalmente recoberto. Para um aumento do contato efetivo matriz-substrato seria necessária a adição de partículas ainda mais finas (nanométricas) ao sistema.

Figura 7 - Simulação do recobrimento da superfície do bloco cerâmico obtido pela interferometria a laser por uma matriz monodispersa constituída pelo cimento $(\mathrm{D} 10=2,87 \mu \mathrm{m})$

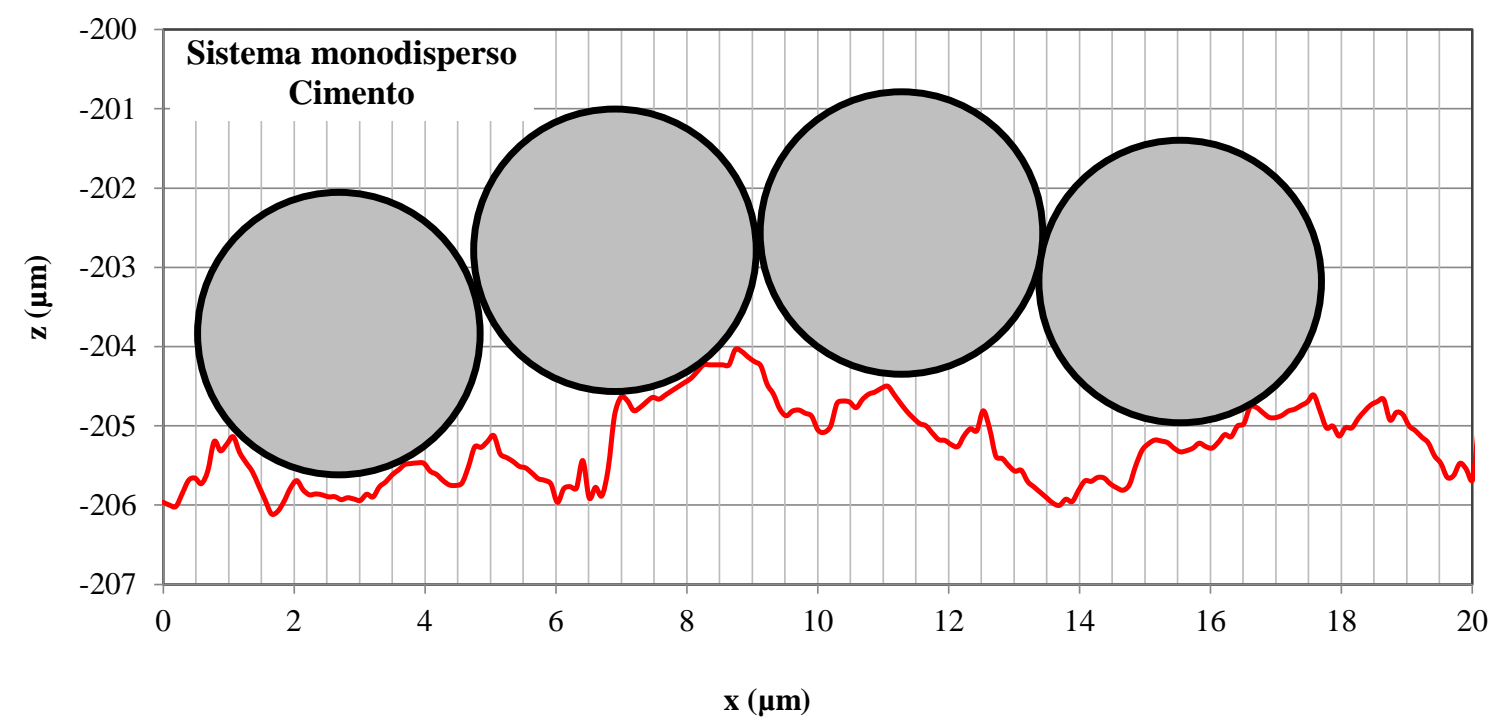

Nota: as partículas não preenchem todas as irregularidades da superfície do substrato.

Figura 8 - Simulação do recobrimento da superfície do bloco cerâmico por uma matriz polidispersa constituída pelo cimento + fino calcário FC1 $(\mathrm{D} 10=1,39 \mu \mathrm{m})$

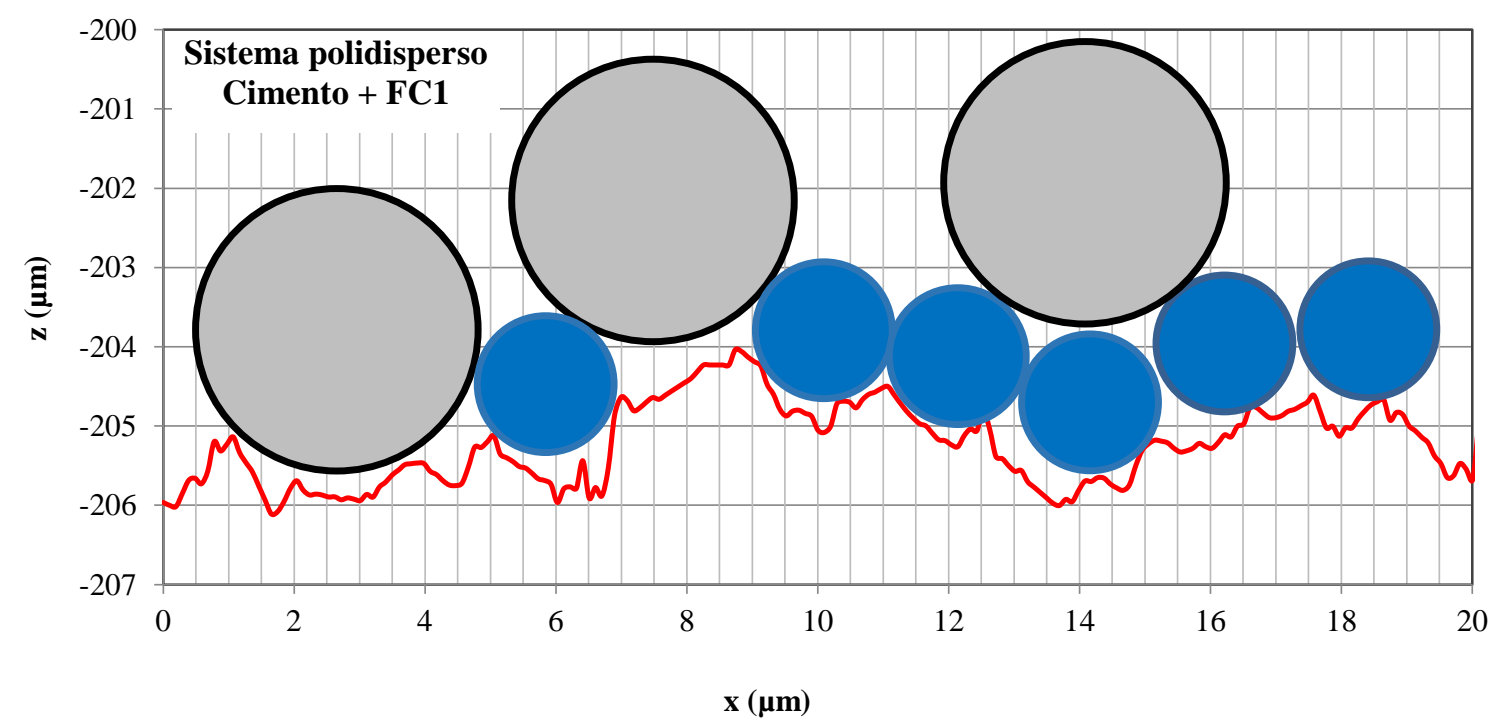

Nota: há aumento do contato efetivo com o uso de FC1, porém a topografia do substrato ainda não é toda recoberta, tornando-se necessária a inclusão de partículas mais finas. 
A aderência argamassa-substrato é controlada pelas forças de Van der Waals entre superfícies em contato: partícula-substrato e partícula-partícula (KENDALL, 2001), independentemente de serem hidratadas ou não (CHATTERJI, 1974). Parece que, no caso da interface, o efeito de diluição do ligante por partículas mais finas e quase inertes resulta numa região mais densa, em que os finos inertes e as fases hidratadas são mais fortemente ligados entre si e ao substrato. Segundo Chatterji, essas forças ocorrem sempre que as partículas estão suficientemente próximas umas das outras, não havendo necessidade de continuidade do arranjo atômico (CHATTERJI, 1974). Isso parece ser válido, dentro de alguns limites, para misturas de partículas inertes com fases hidratadas e superfícies.

Embora seja comum encontrar na literatura referências de correlações diretas entre a resistência de aderência à tração e as propriedades mecânicas das argamassas (CARASEK, 1996), não foi obtida uma correlação entre a resistência de aderência à tração média dos sistemas e as propriedades mecânicas da argamassa, módulo de elasticidade e resistência à tração, como mostram as Figuras 9 e 10 respectivamente. Os resultados indicam que a substituição do ligante por finos calcários e dispersante afetam a resistência da interface diferentemente das propriedades mecânicas. As últimas mostraram-se mais sensíveis à ligeira incorporação de ar das argamassas com dispersante e ao efeito de diluição proporcionado pelo acréscimo do teor de fino calcário. Este resultado está de acordo com Antunes (2005). Em concretos, também não há um consenso sobre a relação da resistência de aderência da interface e a resistência à compressão do concreto (ALEXANDER, 1995). Avanços no entendimento na formação da interface e em metodologias para avaliação dessa região podem contribuir para a otimização tanto da interface como das propriedades mecânicas dos materiais cimentícios.

A resistência de aderência na interface argamassasubstrato é cerca de 4 a 13 vezes inferior à resistência à tração das argamassas estudadas. Essa relação indica a magnitude em que a aderência pode ser melhorada.

\section{Considerações finais}

A substituição do ligante por fino calcário e o uso de dispersantes afetam a aderência de forma diferente das propriedades mecânicas. Há redução dos valores do módulo de elasticidade e resistência à tração da argamassa pela substituição do ligante por fino calcário devido ao efeito de diluição e da adição dispersante pela incorporação de ar. Não foram obtidas correlações entre as propriedades mecânicas da argamassa e a resistência de aderência à tração do sistema argamassa-substrato cerâmico.

Figura 9 - Relação entre a resistência de aderência à tração bloco cerâmico-argamassa e a resistência à tração das argamassas

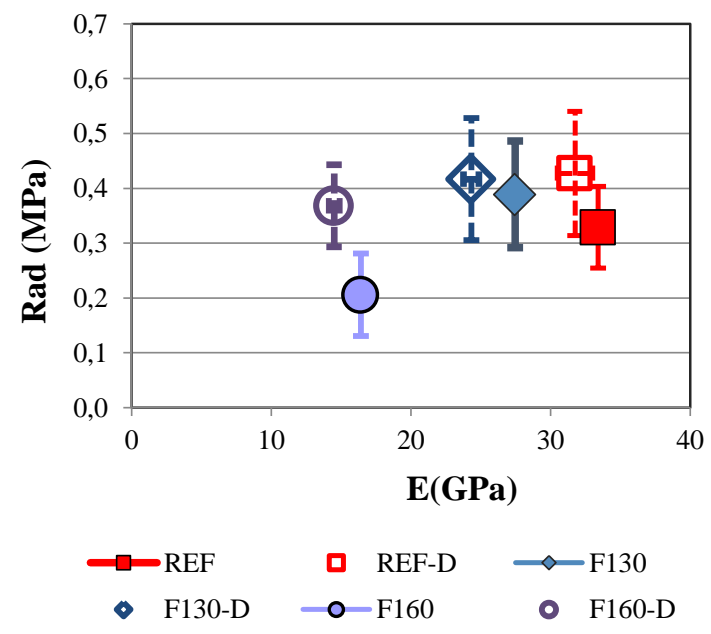


Figura 10 - Relação entre a resistência de aderência à tração bloco cerâmico-argamassa e o módulo de elasticidade das argamassas

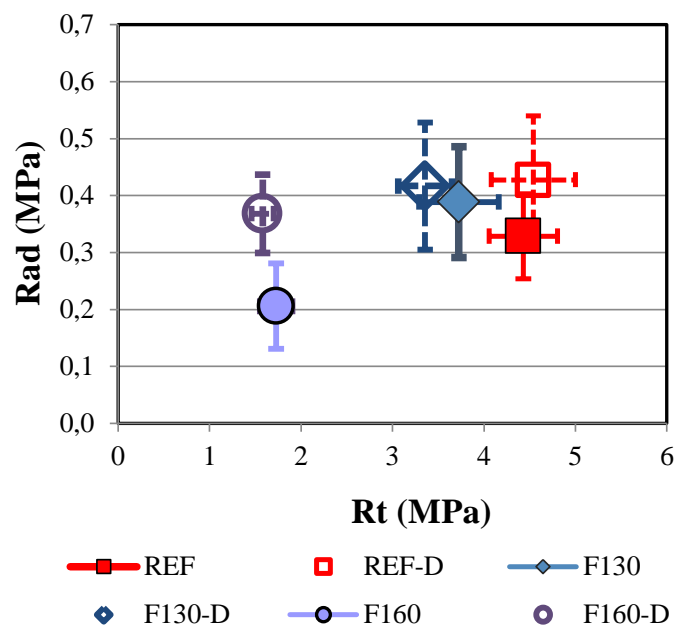

A substituição do ligante em até $30 \%$ por fino calcário com distribuição granulométrica inferior à do ligante não interfere nos valores de aderência. Em teores mais elevados, uma redução é observada. Nesses teores, torna-se necessário o uso de dispersante e partículas mais finas. Por exemplo, a argamassa F160 com dispersante aumentou em $12 \%$ a resistência de aderência em relação à referência sem dispersante e em $76 \%$ quando comparado à mesma sem dispersante. Isso porque as partículas mais finas, se dispersas, aumentam a capacidade de recobrimento da superfície do substrato. Vale ressaltar que o uso de finos em teores elevados requer cuidados, pois pode resultar em retração da argamassa e fissuração. Neste trabalho, utilizou-se cura úmida $(\mathrm{UR}=100 \%)$ e eliminou-se a absorção do substrato.

Os resultados obtidos neste programa experimental mostraram que é possível aumentar a eficiência do uso de ligante através da otimização da granulometria da matriz, acréscimo de finos e dispersão deles, sem reduzir a aderência substratoargamassa. Tais resultados também podem ser aplicados para melhorar a interface de compósitos cimentícios e são úteis para a mitigação de $\mathrm{CO}_{2}$ na cadeia produtiva da indústria de cimento.

\section{Referências}

ALEXANDER, M. G. The Effects of ageing on the Interfacial Zone in Concrete. In: MASO, J. C. (Ed.). Interfacial Trasition Zone in Concrete. 1995. Rilem Report, 11.
AMERICAN SOCIETY FOR TESTING AND MATERIALS. ASTM D3663-03: standard test methods for surface area of catalysts and catalyst carriers. Pennsylvania, 2015.

ANTUNES, R. P. Influência da Reologia e da Energia de Impactonaresistência de aderência de Revestimentos de Argamassa. São Paulo, 2005. 162f. Tese (Doutorado em Engenharia Civil) - Escola Politécnica, Universidade de São Paulo, São Paulo, 2005.

\section{ASSOCIAÇÃO BRASILEIRA DE NORMAS}

TÉCNICAS. NBR 7222: concreto e argamassa: determinação da resistência à tração por compressão diametral de corpos de prova cilindricos. Rio de Janeiro, 2011.

\section{ASSOCIAÇÃO BRASILEIRA DE NORMAS TÉCNICAS. NBR 13278: argamassa para} assentamento e revestimento de paredes e tetos: determinação da densidade de massa e do teor de ar incorporado. Rio de Janeiro, 2005.

\section{ASSOCIAÇÃO BRASILEIRA DE NORMAS}

TÉCNICAS. NBR 13528: revestimento de paredes e tetos de argamassas inorgânicas: determinação da resistência de aderência à tração. Rio de Janeiro, 2010.

\section{ASSOCIAÇÃO BRASILEIRA DE NORMAS \\ TÉCNICAS. NBR 15630: argamassa para} assentamento e revestimento de paredes e tetos: determinação do módulo de elasticidade dinâmico através da propagação de ondaultrassônica. Rio de Janeiro, 2008. 
BARBOSA, W. S. Alteração do Comportamento Reológico da Suspensão Cimentícia Aplicada a Substratos Porosos. São Paulo, 2010. Dissertação (Mestrado em Engenharia Civil) - Escola Politécnica, Universidade de São Paulo, São Paulo, 2010.

BENTZ, D. P. et al. Limestone Fillers to Conserve Cement in Low w/c Concretes: an analysis based on Powers' model. Concrete International, v. 31, 2009.

CAMILETTI, J.; SOLIMAN, A. M.; NEHDI, M. L. Effects of Nano- and Micro-Limestone Addition on Early-age Properties of Ultra-High-

Performance Concrete. Materials and Structures, p. 1-18, oct. 2012.

CARASEK, H. Aderência de Argamassas à Base de Cimento Portland a Substratoporosos: avaliação dos fatoresintervenientes e contribuiçãoaoestudo do mecanismo de ligação. São Paulo, 1996. 285 f. Tese (Doutorado em Engenharia Civil) - Escola Politécnica, Universidade de São Paulo, São Paulo, 1996.

CARVALHO JUNIOR, A. N. Avaliação da Aderência dos Revestimentos Argamassados: umacontribuição à identificação do sistema de aderênciamecânico. Belo Horizonte, 2005. Tese (Doutorado em Engenharia Civl) - Escola de Engenharia, Universidade Federal de Minas Gerais, Belo Horizonte, 2005.

CHATTERJI, S. Load-Bearing Structures and Crystal Intergrowth. Nature, v. 252, p. 383, 1974.

COSTA, E. B. C. Análise de Parâmetros Influentes na Aderência de Matrizes Cimentícias. São Paulo, 2014. 156f. Tese (Doutorado em Engenharia Civil) - Escola Politécnica, Universidade de São Paulo, São Paulo, 2014.

COSTA, E. B. C. Investigação Dométodo de Ensaio de Determinação da Resistência de Aderência de Revestimentos de Argamassa. Goiânia, 2007. 205 f. Dissertação (Mestrado em Engenharia Civil) - Escola de Engenharia, Universidade Federal de Goiás, Goiânia, 2007.

DAMIDOT, D. et al. Thermodynamics and Cement Science. Cement and Concrete Research, v. 41, n. 7, p. 679-695, 2011.

DE FRANÇA, M. S. Comportamento de Mistura de Sistemas Cimentícios Multifásicos Reativos. São Paulo, 2013. Dissertação (Mestrado em Engenharia Civil) - Escola Politécnica, Universidade de São Paulo, São Paulo, 2013.
DÉTRICHÉ, C. H. et al. Mouvements D'eau, Hydration et Comportement Mécanique Des Mortiers D'Enduit. Matériaux et Constructions, v. 17, p. 297-302, 1984.

DÉTRICHÉ, C.; MASO, J. C. Differential Hydration in Rendering Mortars. Cement and Concrete Research, v. 16, n. 3, p. 429-439, 1986.

FIORITO, A. J. S. I. Manual de Argamassas e Revestimentos: estudos e procedimentos de execução. 2. ed. São Paulo: Pini, 2009.

HENDRICKX, R. The Adequate Measurement of the Workability of Masonry Mortar. Belgium, 2009. Tese (Doutorado) - Katholieke Universiteit Leuven, Belgium, 2009.

HU, J.; STROEVEN, P. Properties of the Interfacial Transition Zone in Model Concrete. Interface Science, v. 12, n. 4, p. 389-397, 2004.

HUNGER, M.; BROUWERS, H. J. H. Flow Analysis of Water-Powder Mixtures: application to specific surface area and shape factor. Cement and Concrete Composites, v. 31, p. 39-59, 2009.

INTERNATIONAL ORGANIZATION FOR STANDARDIZATION. ISO 29581: cement: test methods: part 2: chemical analysis by X-ray florescence. 2009.

KENDALL, K. Molecular Adhesion and Its Applications: the sticky universe. Kluwer Academic/Plenum, 2001.

LAWRENCE, P.; CYR, M.; RINGOT, E. Mineral Admixtures in Mortars: effect of inert materials on short-term hydration. Cement and Concrete Research, v. 33, n. 12, p. 1939-1947, 2003.

MATSCHEI, T.; LOTHENBACH, B.; GLASSER, F. The Role of Calcium Carbonate in Cement Hydration. Cement and Concrete Research, v. 37, n. 4, p. 551-558, 2007.

MOOSBERG-BUSTNES, H.; LAGERBLAD, B.; FORSSBERG, E. The Function of Fillers in Concrete. Materials and Structures, v. 37, n. 2, p. 74-81, 2004.

NEHDI, M. Optimization of High Strength Limestone Filler Cement Mortars. Cement and Concrete Research, v. 26, n. 6, p. 883-893, 1996.

OLLIVIER, J. P.; MASO, J. C.; BOURDETTE, B. Interfacial Transition zone in concrete. Advanced Cement Based Materials, v. 2, n. 1, p. 20-30, 1995. 
REDA TAHA, M. M.; EL-DIEB, A. S.; SHRIVE, N. G. Sorptivity: a reliable measurement for surface absorption of masonry brick units.

Materials and Structures, v. 34, n. 7, p. 438-445, 2005.

ROCCO, C. et al. Size Effect and Boundary Conditions in the Brazilian Test: experimental verification. Materials and Structures, v. 32, p. 210-217, apr. 1999.

ROY, D. M. et al. Cement Paste Aggregate Interface Microstructure. National Academy of Sciences, v. 79, 1993.

SCRIVENER, K. L.; CRUMBIE, A. K.; LAUGESEN, P. The Interfacial Transition Zone (ITZ) Between Cement Paste and Aggregate in Concrete. Interface Science, v. 12, n. 4, p. 411 421, 2004.
TSIVILIS, S. et al. Properties and Behavior of Limestone Cement Concrete and Mortar. Cement and Concrete Research, v. 30, n. 10, p. 16791683,2000

UCHIKAWA, H.; HANEHARA, S.; SAWAKI, D. The Role of Steric Repulsive Force in the Dispersion of Cement Particles In the Fresh Paste Prepared with Organic Admixture. Cement and Concrete Research, v. 27, n. 1, p. 37-50, 1997.

\section{Agradecimentos}

Os autores gostariam de agradecer o apoio financeiro da CAPES, CNPq, FAPESP (Processos $\mathrm{n}^{\circ}$ 2010/10843-7 e $\mathrm{n}^{\circ}$ 2010/51284-0); ao CONSITRA - Consórcio Setorial para Inovação Tecnológica em Revestimentos de Argamassa pelo apoio e a P\&D ANEEL pelo Projeto de Reologia 394-0929/2010 desenvolvido em parceria pela FUSP e FURNAS.

\section{Eliane Betânia Carvalho Costa}

Departamento Acadêmico de Construção Civil | Universidade Tecnológica Federal do Paraná | Rua Deputado Heitor Alencar Furtado, 4001/4002, Cidade Industrial | Curitiba - PR - Brasil | CEP 81280-340 | Tel.: (41) 3279-4543 | E-mail: elianecosta@utfpr.edu.br

\section{Fábio Alonso Cardoso}

Departamento de Engenharia de Construção Civil, Escola Politécnica | Universidade de São Paulo | Av. Prof. Almeida Prado, trav. 2, n. 83, Cidade Universitária | São Paulo - SP - Brasil | CEP 05508-900 | Tel.: (11) 3091-5248 Ramal 5248 |

E-mail: fabio.cardoso@lme.pcc.usp.br

\section{Vanderley Moacyr John}

Departamento de Engenharia de Construção Civil, Escola Politécnica | Universidade de São Paulo | Tel.: (11) 3091-5794 |

E-mail: vmjohn@lme.pcc.usp.br

Revista Ambiente Construído

Associação Nacional de Tecnologia do Ambiente Construído

Av. Osvaldo Aranha, $99-3^{\circ}$ andar, Centro

Porto Alegre - RS - Brasil

CEP $90035-190$

Telefone: +55 (51) 3308-4084

Fax: +55 (51) 3308-4054

www.seer.ufrgs.br/ambienteconstruido

E-mail: ambienteconstruido@ufrgs.br 